\title{
Impulsive Controller Design for Complex Nonlinear Singular Networked Systems with Packet Dropouts
}

\author{
Xian-Lin Zhao, ${ }^{1,2}$ Ai-Min Wang, ${ }^{1}$ Cun-Li Dai, ${ }^{2}$ Xiao-Lin Li, ${ }^{2}$ and Wei Lu \\ ${ }^{1}$ School of Instrument Science and Engineering, Southeast University, Nanjing 210096, China \\ ${ }^{2}$ College of Engineering, Nanjing Agricultural University, Nanjing 210031, China \\ Correspondence should be addressed to Cun-Li Dai; daicunli@163.com
}

Received 12 September 2013; Accepted 14 October 2013

Academic Editor: Tao Li

Copyright (c) 2013 Xian-Lin Zhao et al. This is an open access article distributed under the Creative Commons Attribution License, which permits unrestricted use, distribution, and reproduction in any medium, provided the original work is properly cited.

Globally exponential stability of Complex (with coupling) Nonlinear Singular Impulsive Networked Control Systems (CNSINCS) with packet dropouts and time-delay is investigated. Firstly, the mathematic model of CNSINCS is established. Then, by employing the method of Lyapunov functional, exponential stability criteria are obtained and the impulsive controller design method is given. Finally, some simulation results are provided to demonstrate the effectiveness of the proposed method.

\section{Introduction}

At present, singular system is widely used in the control of spacecraft, flexible robot, complex power, large chemical and wireless transmission system [1-5]. Many results had been achieved for discrete singular system and time-delay singular system. Such as in [6], the nonlinear discrete singular perturbation model was established and the system condition was given. In [7], chattering free sliding mode control for uncertain discrete time-delay singular system was investigated. The asymptotically stable was established, and the chattering problem that appears in traditional variable structure system was eliminated. As for time-delay singular system, the stability of uncertain time-delay singular systems was researched and the asymptotic stability condition was achieved in [8] by using Jensen integral inequality and feedback control method.

On the other hand, singular system has impulsive behavior in many cases [9-14]. So it is very important to discuss the problem of impulsive control. For the stability of the impulsive control system, nonlinear impulsive control was put forward and the concept of asymptotic stability condition was provided in [11]. Asymptotic stability condition for a class of uncertain impulsive system was established through the comparison theorem in [12]. Switch control method was used to research the stability of singular impulsive system, robust stabilization, and $H_{\infty}$ control problem in [13]. Linear approximation and the LMI method were used, respectively, to study the problem of system stability and the sufficient conditions for asymptotic stability in [14].

In network impulsive control system packet dropouts and time-delay exist which will influence the stability of singular system. It is necessary to analyze stability condition and the method of controller design. That is the problem focussed in this study. According to the Lyapunov function theory and comparison theorem, the sufficient conditions for the global exponential stability of the system is obtained. The detailed design process of impulsive controller is given in the paper. System will be stable in accordance with the decay rate to achieve exponential stability. A numerical example is provided to illustrate the correctness of theoretical and the effectiveness of design method.

\section{The Mathematic Model of CNSINCS}

The mathematic model of CNSINCS can be described as

$$
\begin{array}{r}
E \dot{x}_{i}(t)=A x_{i}(t)+f\left(x_{i}(t)\right)+\sum_{j=1}^{N} G_{i j} \Gamma x_{j}(t-\tau(t)), \\
i=1, \ldots, N, t \in\left(t_{k-1}, t_{k}\right]
\end{array}
$$




$$
\begin{gathered}
x_{i}\left(t_{k}^{+}\right)=c_{i} x_{i}\left(t_{k}\right)+\bar{u}_{i}\left(t_{k}\right), \quad t=t_{k}^{+}, \quad k=1,2, \ldots \\
x_{i}(t)=\varphi_{i}(t), \quad t \in[-\bar{\tau}, 0]
\end{gathered}
$$

where $x_{i}(t) \in R^{n}$ is the state vector of the $i$ th node. $A$ is a constant matrix of $n \times n$. $c_{i}$ is known scalar. $E \in R^{n \times n}$ is a singular constant matrix, and $0<\operatorname{rank} E=r \leq n$, without loss of generality; we hypotheses $E=\left[\begin{array}{cc}I_{r} & 0 \\ 0 & 0\end{array}\right] . f(\cdot)$ is a nonlinear function. $\Gamma$ is the internal coupling matrix. $G=G_{i j} \in R^{n \times n}$ is the coupling matrix of the whole network structure and weights. $\tau(t)$ is network transmission delay and is assumed to satisfy $0 \leq \tau(t) \leq \bar{\tau}$.

In the process of data transmitting, the buffer's model can be described as:

$$
\begin{aligned}
& \bar{u}_{i}\left(t_{k}\right)= \begin{cases}u_{i}\left(t_{k}\right), & k=1,2, \ldots, \text { if transmitted successfully, } \\
\bar{u}_{i}\left(t_{k-1}\right), & k=1,2, \ldots, \text { otherwise, }\end{cases} \\
& \bar{x}_{i}\left(t_{k}\right)= \begin{cases}x_{i}\left(t_{k}\right), & k=1,2, \ldots, \text { if transmitted successfully, } \\
\bar{x}_{i}\left(t_{k-1}\right), & k=1,2, \ldots, \text { otherwise. }\end{cases}
\end{aligned}
$$

The impulsive controller can be designed as

$$
\bar{u}_{i}\left(t_{k}\right)=K_{i} \bar{x}_{i}\left(t_{k}\right),
$$

where $\bar{u}_{i}\left(t_{k}\right) \in R^{m}$. Substituting (2) and (3) into (1), the closed-loop nonlinear singular impulsive networked system model is obtained as follows:

$$
\begin{gathered}
E \dot{x}_{i}(t)=A x_{i}(t)+f\left(x_{i}(t)\right)+\sum_{j=1}^{N} G_{i j} \Gamma x_{j}(t-\tau(t)), \\
i=1, \ldots, N, t \in\left(t_{k-1}, t_{k}\right], \\
x_{i}\left(t_{k}^{+}\right)=c_{i} x_{i}\left(t_{k}\right)+\left(1-\sigma_{i}\left(t_{k}\right)\right) K_{i} x_{i}\left(t_{k}\right) \\
+\sigma_{i}\left(t_{k}\right) K_{i} \bar{x}_{i}\left(t_{k-1}\right), \quad t=t_{k}^{+}, k=1,2, \ldots, \\
x_{i}(t)=\varphi_{i}(t), \quad t \in[-\bar{\tau}, 0],
\end{gathered}
$$

where $\sigma_{i}\left(t_{k}\right)=1$ denotes that there are data dropouts and, $\sigma_{i}\left(t_{k}\right)=0$, there are no packet dropouts.

Lemma 1. Let $P \in R^{n \times n}$ be a symmetric positive definite matrix and $P=Q^{T} Q$. For any $x, y \in R^{n \times n}$ and $A \in R^{n \times n}$, then

(1) $x^{T}\left(A^{T} P E+E^{T} P^{T} A\right) x \leq 2 \lambda_{\max }(A) x^{T} E^{T} P E x$,

(2) $x^{T}\left(A^{T} P+P A\right) x \leq 2 \mu\left(Q A Q^{-1}\right) x^{T} P x$,

(3) $\left|x^{T} P y\right| \leq \sqrt{x^{T} P x} \sqrt{y^{T} P y}$.

Lemma 2. According to the definition of Kronecker product, for a given matrix $A, B$, and scalar $\alpha$, the following equality can be achieved:

(1) $(\alpha A) \otimes B=A \otimes(\alpha B)$,

(2) $(A+B) \otimes C=(A \otimes C)+(B \otimes C)$,

(3) $(A \otimes B) \otimes(C \otimes D)=(A C) \otimes(B D)$.
If $X(t)=\left(x_{1}^{T}(t), \ldots, x_{N}^{T}(t)\right)^{T}, C=\operatorname{diag}\left\{c_{1}, \ldots, c_{N}\right\}$, and $F(X(t))=\left(f^{T}\left(x_{1}^{T}(t), \ldots, f^{T}\left(x_{N}^{T}(t)\right)^{T}\right.\right.$, according to Lemma 1, the complex nonlinear singular system can be expressed as

$$
\begin{aligned}
\left(I_{N} \otimes E\right) \dot{X}(t)= & \left(I_{N} \otimes A\right) X(t)+F(X(t)) \\
& +(G \otimes \Gamma) X(t-\tau(t)), \quad t \in\left(t_{k-1}, t_{k}\right], \\
X\left(t_{k}^{+}\right)= & C X\left(t_{k}\right)+\left(1-\sigma\left(t_{k}\right)\right) K X\left(t_{k}\right) \\
& +\sigma\left(t_{k}\right) \bar{X}\left(t_{k-1}\right), \quad t=t_{k}^{+} .
\end{aligned}
$$

Suppose

$$
\begin{aligned}
\bar{E}=\left[\begin{array}{ll}
E & 0 \\
0 & 0
\end{array}\right], & \bar{I}_{N}=\left[\begin{array}{cc}
I_{N} & 0 \\
0 & 0
\end{array}\right], \quad Z\left(t_{k}\right)=\left[\begin{array}{c}
X\left(t_{k}\right) \\
X\left(t_{k-1}\right)
\end{array}\right], \\
\bar{A} & =\left[\begin{array}{cc}
A & 0 \\
0 & 0
\end{array}\right] .
\end{aligned}
$$

Then (5) is equivalent to the following system:

$$
\begin{aligned}
& \left(\bar{I}_{N} \otimes \bar{E}\right) \dot{z}(t)=\left(\bar{I}_{N} \otimes \bar{A}\right) z(t)+\left[\begin{array}{c}
F(x(t)) \\
0
\end{array}\right] \\
& +\left[\begin{array}{cc}
G \otimes \Gamma & 0 \\
0 & 0
\end{array}\right] z(t-\tau(t)) \\
& \left(\bar{I}_{N} \otimes \bar{E}\right) z\left(t_{k}^{+}\right) \\
& =C\left(\bar{I}_{N} \otimes \bar{E}\right) z\left(t_{k}\right) \\
& \quad+\left[\begin{array}{cc}
\left(1-\sigma\left(t_{k}\right)\right) K & \sigma\left(t_{k}\right) K \\
0 & 0
\end{array}\right]\left(\bar{I}_{N} \otimes \bar{E}\right) z\left(t_{k}\right) .
\end{aligned}
$$

Lemma 3 (see [15]). If $L(t, z(t))$ and $U_{k}(z(t))$ satisfy the Lipchitz condition, there exists a uniqueness of solution to nonlinear singular impulsive differential equation which is written as

$$
\begin{gathered}
\dot{z}(t)=L(x, t), \quad t \in\left(t_{k-1}, t_{k}\right], \\
\Delta z(t)=U_{k}(z(t)), \quad t=t_{k}^{+}, k=1,2, \ldots,
\end{gathered}
$$

where $z(t) \in R^{n}, L: R_{+} \times R^{n} \rightarrow R^{n}, U_{k}: R^{n} \rightarrow R^{n}$.

\section{The Design of CNSINCS}

For the nonlinear singular networked impulsive control system (4), we have the following theorem. 
Theorem 4. If there exist $0<\rho=\sup _{k \in N}\left\{t_{k}-t_{k-1}\right\}<\infty$ and a nonsingular matrix $K \in R^{n \times n}$, such that

$$
\begin{aligned}
\frac{2 \ln \beta}{\rho}+ & \left(2 \lambda_{\max }(\bar{A})+2 L \frac{\lambda_{\max }\left(\left(\bar{I}_{N} \otimes \bar{E}\right)^{T} P\right)}{\lambda_{\min }\left(\left(\bar{I}_{N} \otimes \bar{E}\right)^{T} P\right)}+1\right) \\
+\lambda_{\max }^{2}(G \otimes \Gamma) & <1 \\
0 & <\beta<1,
\end{aligned}
$$

where

$$
\beta=\left[\lambda_{\max }^{2}\left(C+\left[\begin{array}{cc}
\left(1-\sigma\left(t_{k}\right)\right) K & \sigma\left(t_{k}\right) K \\
0 & 0
\end{array}\right]\right)\right]^{1 / 2}
$$

then the nonlinear singular networked impulsive control system (4) is asymptotically stable:

$$
\|x(t)\| \leq \frac{1}{\beta} \sqrt{\frac{\lambda_{\max }(P)}{\lambda_{\min }(P)}} \sup _{-\tau \leq \bar{\theta} \leq 0}\{\|\phi(\theta)\|\} e^{-(\lambda / 2) t},
$$

where $\lambda$ is the positive solution of $\lambda+p+q e^{\lambda \bar{\tau}}=0$, and $q>$ $0, p+q<0$ :

$$
\begin{aligned}
p= & \frac{2 \ln \beta}{\rho}+\left(2 \lambda_{\max }(\bar{A})+2 L \frac{\lambda_{\max }\left(\left(\bar{I}_{N} \otimes \bar{E}\right)^{T} P\right)}{\lambda_{\min }\left(\left(\bar{I}_{N} \otimes \bar{E}\right)^{T} P\right)}+1\right) \\
& +\lambda_{\max }^{2}(G \otimes \Gamma) .
\end{aligned}
$$

Proof. From [16], we know that $\lambda+p+q e^{\lambda \bar{\tau}}=0$ must have a solution. Set $V(t)=z^{T}(t)\left(\bar{I}_{N} \otimes \bar{E}\right)^{T} P\left(\bar{I}_{N} \otimes \bar{E}\right) z(t)$, where $\left(\bar{I}_{N} \otimes \bar{E}\right)^{T} P\left(\bar{I}_{N} \otimes \bar{E}\right) \geq 0$.

When $t \in\left(t_{k-1}, t_{k}\right]$, the derivative of $V(t)$ along the trajectories of the CNSINCS (4) is

$$
\begin{aligned}
\dot{V}(t)= & \left(\left(\bar{I}_{N} \otimes \bar{E}\right) \dot{z}(t)\right)^{T} P\left(\bar{I}_{N} \otimes \bar{E}\right) z(t) \\
& +\left(\left(\bar{I}_{N} \otimes \bar{E}\right) z(t)\right)^{T} P^{T}\left(\bar{I}_{N} \otimes \bar{E}\right) \dot{z}(t) \\
= & \left(\left(\bar{I}_{N} \otimes \bar{A}\right) z(t)+\left[\begin{array}{c}
F(x(t)) \\
0
\end{array}\right]+\left[\begin{array}{cc}
G \otimes \Gamma & 0 \\
0 & 0
\end{array}\right]\right. \\
& \times z(t-\tau(t)))^{T} P\left(\bar{I}_{N} \otimes \bar{E}\right) z(t) \\
& +\left(\left(\bar{I}_{N} \otimes \bar{E}\right) z(t)\right)^{T} P\left(\bar{I}_{N} \otimes \bar{A}\right) z(t)\left[\begin{array}{c}
F(x(t)) \\
0
\end{array}\right] \\
& +\left[\begin{array}{cc}
G \otimes \Gamma & 0 \\
0 & 0
\end{array}\right] z(t-\tau(t))
\end{aligned}
$$

$$
\begin{aligned}
= & z^{T}(t)\left(\bar{A}^{T} P\left(\bar{I}_{N} \otimes \bar{E}\right)+\left(\bar{I}_{N} \otimes \bar{E}\right)^{T} P^{T} \bar{A}\right) \\
& \times z(t)+F^{T}(x(t)) P\left(\bar{I}_{N} \otimes \bar{E}\right) z(t) \\
& +z^{T}(t)\left(\bar{I}_{N} \otimes \bar{E}\right)^{T} P F(x(t)) \\
& +z^{T}(t-\tau(t)) G^{T} P E z(t) \\
& +z^{T}(t)\left(\bar{I}_{N} \otimes \bar{E}\right)^{T} P^{T} G z(t-\tau(t)) .
\end{aligned}
$$

According to Lemma 3 we have

$\dot{V}(t)$

$$
\begin{aligned}
\leq & 2 \lambda_{\max }(\bar{A}) z^{T}(t)\left(\bar{I}_{N} \otimes \bar{E}\right)^{T} P\left(\bar{I}_{N} \otimes \bar{E}\right) z(t) \\
& +2 L \lambda\left(\bar{I}_{N} \otimes \bar{E}\right)^{T} P\|z(t)\| \\
& +2 \sqrt{z^{T}(t) P\left(\bar{I}_{N} \otimes \bar{E}\right) z(t)} \\
& \times \sqrt{z^{T}(t-\tau(t))(G \otimes \Gamma)^{T}\left(\bar{I}_{N} \otimes \bar{E}\right)^{T} P(G \otimes \Gamma) z(t-\tau(t))} \\
\leq & \lambda_{\max }^{2}(G \otimes \Gamma) z^{T}(t-\tau(t))\left(\bar{I}_{N} \otimes \bar{E}\right)^{T} P\left(\bar{I}_{N} \otimes \bar{E}\right) z(t-\tau(t)) \\
& +2 L \frac{\lambda_{\max }\left(\left(\bar{I}_{N} \otimes \bar{E}\right)^{T} P\right)}{\lambda_{\min }\left(\left(\bar{I}_{N} \otimes \bar{E}\right)^{T} P\right)} z^{T}(t)\left(\bar{I}_{N} \otimes \bar{E}\right)^{T} P\left(\bar{I}_{N} \otimes \bar{E}\right) z(t) \\
& +2 \lambda_{\max }(\bar{A}) z^{T}(t)\left(\bar{I}_{N} \otimes \bar{E}\right)^{T} P\left(\bar{I}_{N} \otimes \bar{E}\right) z(t) \\
& +z^{T}(t)\left(\bar{I}_{N} \otimes \bar{E}\right)^{T} P\left(\bar{I}_{N} \otimes \bar{E}\right) z(t) .
\end{aligned}
$$

On the other hand, when $t=t_{k}^{+}$,

$$
\begin{aligned}
\dot{V}\left(t_{k}^{+}\right)= & z^{T}\left(t_{k}^{+}\right)\left(\bar{I}_{N} \otimes \bar{E}\right)^{T} P\left(\bar{I}_{N} \otimes \bar{E}\right) z\left(t_{k}^{+}\right) \\
= & z^{T}\left(t_{k}^{+}\right)\left(\bar{I}_{N} \otimes \bar{E}\right)^{T}\left(\bar{I}_{N} \otimes \bar{E}\right)^{T} \\
& \times P\left(\bar{I}_{N} \otimes \bar{E}\right)\left(\bar{I}_{N} \otimes \bar{E}\right) z\left(t_{k}^{+}\right) \\
= & {\left[\left(\bar{I}_{N} \otimes \bar{E}\right) z^{T}\left(t_{k}^{+}\right)\right]^{T}\left(\bar{I}_{N} \otimes \bar{E}\right)^{T} P\left(\bar{I}_{N} \otimes \bar{E}\right) } \\
& \times\left[\left(\bar{I}_{N} \otimes \bar{E}\right) z\left(t_{k}^{+}\right)\right] \\
= & \left(C\left(\bar{I}_{N} \otimes \bar{E}\right) z\left(t_{k}\right)\right. \\
& \left.+\left[\begin{array}{cc}
\left(1-\sigma\left(t_{k}\right)\right) K & \sigma\left(t_{k}\right) K \\
0 & 0
\end{array}\right]\left(\bar{I}_{N} \otimes \bar{E}\right) z\left(t_{k}\right)\right)^{T} \\
\left(\bar{I}_{N} \otimes \bar{E}\right)^{T} P\left(\bar{I}_{N} \otimes \bar{E}\right) & \\
& \times\left(C+\left[\begin{array}{cc}
\left(1-\sigma\left(t_{k}\right)\right) K & \sigma\left(t_{k}\right) K \\
0 & 0
\end{array}\right]\right)\left(\bar{I}_{N} \otimes \bar{E}\right) z\left(t_{k}\right)
\end{aligned}
$$




$$
\begin{aligned}
& =z^{T}\left(t_{k}\right)\left(\bar{I}_{N} \otimes \bar{E}\right)^{T} \\
& \times\left(C+\left[\begin{array}{cc}
\left(1-\sigma\left(t_{k}\right)\right) K & \sigma\left(t_{k}\right) K \\
0 & 0
\end{array}\right]\right)^{T} \\
& \times\left(\bar{I}_{N} \otimes \bar{E}\right)^{T}, \\
& P\left(\bar{I}_{N} \otimes \bar{E}\right) z\left(t_{k}\right) \\
& \leq \lambda_{\max }^{2}\left(C+\left[\begin{array}{cc}
\left(1-\sigma\left(t_{k}\right)\right) K & \sigma\left(t_{k}\right) K \\
0 & 0
\end{array}\right]\right) \\
& \times z^{T}\left(t_{k}\right)\left(\bar{I}_{N} \otimes \bar{E}\right)^{T}\left(\bar{I}_{N} \otimes \bar{E}\right)^{T} P, \\
& \left(\bar{I}_{N} \otimes \bar{E}\right)\left(\bar{I}_{N} \otimes \bar{E}\right) z\left(t_{k}\right) \\
& =\lambda_{\max }^{2}\left(C+\left[\begin{array}{cc}
\left(1-\sigma\left(t_{k}\right)\right) K & \sigma\left(t_{k}\right) K \\
0 & 0
\end{array}\right]\right) \\
& \times z^{T}\left(t_{k}\right)\left(\bar{I}_{N} \otimes \bar{E}\right)^{T} P\left(\bar{I}_{N} \otimes \bar{E}\right) z\left(t_{k}\right) \\
& =\beta^{2} V\left(t_{k}\right) \text {, }
\end{aligned}
$$

where $\beta^{2}=\lambda_{\max }^{2}\left(C+\left[\begin{array}{cc}\left(1-\sigma\left(t_{k}\right)\right) K & \sigma\left(t_{k}\right) K \\ 0\end{array}\right]\right)$, supposing that $\varepsilon>0$ is random constant, a comparison system can be established as follows:

$$
\begin{gathered}
\dot{v}(t)=\left(2 \lambda_{\max }(\bar{A})+2 L \frac{\lambda_{\max }\left(\left(\bar{I}_{N} \otimes \bar{E}\right)^{T} P\right)}{\lambda_{\min }\left(\left(\bar{I}_{N} \otimes \bar{E}\right)^{T} P\right)}+1\right) v(t) \\
+\lambda_{\max }^{2}(G \otimes \Gamma) v(t-\tau(t))+\varepsilon \quad t \neq t_{k}, \\
v\left(t_{k}^{+}\right)=\beta v\left(t_{k}\right), \quad t=t_{k}, \\
v(\theta)=\lambda_{\max }(P)\|\phi(\theta)\|^{2}, \quad-\bar{\tau} \leq \theta \leq 0 .
\end{gathered}
$$

It is clear that $V(t) \leq v(t)$ when $-\bar{\tau} \leq \theta \leq 0$, according to [16], and we have $V(t) \leq v(t)$ when $t \geq 0$; the trivial solution of the comparison system is

$$
\begin{aligned}
v(t)= & W(t, 0) v(0) \\
& +\int_{0}^{t} W(t, s) \lambda_{\max }^{2}(G \otimes \Gamma) v(s-\tau(s)+\varepsilon) d s, \quad t \geq 0,
\end{aligned}
$$

where $W(t, s)$ is Cauchy matrix which satisfies

$$
\begin{aligned}
W(t, s)= & \beta^{2 \eta(t, s)} \\
& \times e^{\left\{\left(2 \lambda_{\max }(\bar{A})+2 L\left(\lambda_{\max }\left(\left(\bar{I}_{N} \otimes \bar{E}\right)^{T} P\right) / \lambda_{\min }\left(\left(\bar{I}_{N} \otimes \bar{E}\right)^{T} P\right)\right)+1\right)(t-s)\right\}} \\
& \leq \beta^{2(t-s / \rho)-1} e^{(p-(2 \ln \beta / \rho))(t-s)} \leq \beta^{-2} e^{p(t-s)}
\end{aligned}
$$

in which $\eta(t, s)$ is the number of control impulses in the interval $(s, t], 0<\rho=\sup _{k \in N}\left\{t_{k}-t_{k-1}\right\}<\infty$, for $t \geq 0$; we have

$$
\begin{aligned}
v(t) \leq & \beta^{-2} \lambda_{\max }\left(\left(\bar{I}_{N} \otimes \bar{E}\right)^{T} P\left(\bar{I}_{N} \otimes \bar{E}\right)\right)\|\varphi(0)\|^{2} \\
& +\int_{0}^{t} \beta^{-2} e^{p(t-s)} \lambda_{\max }^{2}(G \otimes \Gamma) v(s-\tau(s)+\varepsilon) d s \\
\leq & \gamma e^{p t}+e^{p(t-s)}(q v(s-\tau(s)+\varepsilon) d s,
\end{aligned}
$$

where

$$
\begin{aligned}
\gamma & =\beta^{-2} \lambda_{\max }\left(\left(\bar{I}_{N} \otimes \bar{E}\right)^{T} P\left(\bar{I}_{N} \otimes \bar{E}\right)\right) \sup _{-\bar{\tau} \leq s \leq 0}\|\varphi(s)\|^{2} \\
& =\beta^{-2} \lambda_{\max }(P) \sup _{-\bar{\tau} \leq s \leq 0}\|\varphi(s)\|^{2} .
\end{aligned}
$$

In the following, we will prove that the following inequality holds:

$$
v(t) \leq \gamma e^{-\lambda t}-\frac{\varepsilon}{\beta^{2} p}, \quad t \geq 0
$$

Since $\varepsilon>0, p<0$, so $\varepsilon / \beta^{2} p<0$. If there exists $t^{*}>0$ which satisfies

$$
v\left(t^{*}\right) \geq \gamma e^{-\lambda t^{*}}-\frac{\varepsilon}{\beta^{2} p}
$$

$$
v(t)>\gamma e^{-\lambda t}-\frac{\varepsilon}{\beta^{2} p}, \quad t<t^{*} .
$$

From (16) and (24) we have

$$
\begin{aligned}
& v\left(t^{*}\right) \\
& \leq \gamma e^{-\lambda t^{*}}+\int_{0}^{t^{*}} e^{p\left(t^{*}-s\right)}[q v(s-\tau(s))+\varepsilon] d s \\
& <e^{p t^{*}}\left\{\gamma-\frac{\varepsilon}{\beta^{2}(p+q)}\right. \\
& \left.\quad+\int_{0}^{t^{*}} e^{-p s}\left[\gamma q e^{-\lambda(s-\tau(s))}-\frac{\varepsilon q}{\beta^{2}(p+q)}+\frac{\varepsilon}{\beta^{2}}\right] d s\right\} \\
& <e^{p t^{*}}\left\{\gamma-\frac{\varepsilon}{\beta^{2}(p+q)}+\gamma q e^{\lambda \tau}\right. \\
& \left.\quad \times \int_{0}^{t^{*}} e^{-(p+\lambda) s} d s+\frac{\varepsilon p}{\beta^{2}(p+q)} \int_{0}^{t^{*}} e^{-p s} d s\right\} \\
& <e^{p t^{*}}\left\{\gamma-\frac{\varepsilon}{\beta^{2}(p+q)}\right\} \\
& \left.+\gamma\left[e^{-(p+\lambda) t^{*}}-1\right]-\frac{\varepsilon}{\beta^{2}(p+q)}\left(e^{-p t^{*}}-1\right)\right\} \\
& =\gamma e^{-\lambda t^{*}}-\frac{\varepsilon}{\beta^{2}(p+q)}
\end{aligned}
$$


which contradicts with (23), and consequently (22) holds. Let $\varepsilon \rightarrow 0$; then

$$
V(t) \leq v(t) \leq \gamma e^{-\lambda t}, \quad t \geq 0
$$

Moreover

$$
V(t) \geq \lambda_{\min }(P)\|x(t)\|^{2}, \quad t \geq 0 .
$$

Combining the inequality (26) and (27),

$$
\|x(t)\| \leq\left(\frac{1}{\beta}\right) \sqrt{\frac{\lambda_{\max }(P)}{\lambda_{\min }(P)}} \sup _{-\tau \leq \bar{\theta} \leq 0}\{\|\phi(\theta)\|\} e^{-(\lambda / 2) t}
$$

which implies conclusion (22) and this completes the proof.

Remark 5. For the case $\beta \geq 1$, we can replace the condition $0<\rho=\sup _{k \in N}\left\{t_{k}-t_{k-1}\right\}<\infty$ with $0<\varsigma=\inf _{k \in N}\left\{t_{k}-t_{k-1}\right\}<$ $\infty$; then the conclusion of Theorem 4 still holds except that now inequality (9) becomes

$$
\begin{aligned}
& \frac{2 \ln \beta}{\varsigma}+\left(2 \lambda_{\max }(\bar{A})+2 L \frac{\lambda_{\max }\left(\left(\bar{I}_{N} \otimes \bar{E}\right)^{T} P\right)}{\lambda_{\min }\left(\left(\bar{I}_{N} \otimes \bar{E}\right)^{T} P\right)}+1\right) \\
& +\lambda_{\max }^{2}(G \otimes \Gamma)<1 .
\end{aligned}
$$

For $\beta \geq 1$, we have

$$
\left\|x_{i}(t)-x_{j}(t)\right\| \leq \frac{1}{\sqrt{\beta}} \sup _{-\bar{\tau} \leq t \leq 0}\{\|\phi(t)\|\} e^{-(\lambda / 2) t}
$$

in which $\lambda=\max _{k \in N}\left\{\ln \beta /\left(t_{k}-t_{k-1}\right)\right\}$.

The proof of the above conclusion remains largely the same as Theorem 4, so we omitted it to avoid repetition.

\section{Design Procedure of Impulsive Control for Complex Network}

According to Theorem 4, the design process of impulsive control is given as follows.

(1) Calculate the parameters $L, m$.

(2) Choose a matrix $P$ which satisfies $\left(\bar{I}_{N} \otimes \bar{E}\right)^{T} P\left(\bar{I}_{N} \otimes \bar{E}\right) \geq$ 0 .

(3) For a given parameter $\lambda_{0}$, we can determine the control sequence $\left\{t_{k}\right\}, t \in N$ as follows. If $0<$ $\beta<1$, let $\Theta:=\left(2 \lambda_{\max }(\bar{A})+2 L\left(\lambda_{\max }\left(\left(\bar{I}_{N} \otimes \bar{E}\right)^{T} P\right) /\right.\right.$ $\left.\left.\lambda_{\min }\left(\left(\bar{I}_{N} \otimes \bar{E}\right)^{T} P\right)\right)+1\right)+\lambda_{\max }^{2}(G \otimes \Gamma)$; then the upper bounds of time can be taken as $0<\rho=\sup _{k \in N}\left\{t_{k}-\right.$ $\left.t_{k-1}\right\}=-(\ln \beta) / \Theta$; if $\beta \geq 1$, let $\Theta:=\left(2 \lambda_{\max }(\bar{A})+\right.$ $\left.2 L\left(\lambda_{\max }\left(\left(\bar{I}_{N} \otimes \bar{E}\right)^{T} P\right) / \lambda_{\min }\left(\left(\bar{I}_{N} \otimes \bar{E}\right)^{T} P\right)\right)+1\right)+$ $\lambda_{\max }^{2}(G \otimes \Gamma)$; then the lower bounds of control intervals is $0<\varsigma=\inf _{k \in N}\left\{t_{k}-t_{k-1}\right\}=(\ln \beta) / \Theta$.

\section{Numerical Simulation}

In this section, a numerical example is presented to illustrate the effectiveness of derived results.

Example 6. Consider the following complicated nonlinear singular system:

$$
\begin{gathered}
E \dot{x}_{i}(t)=A x_{i}(t)+f\left(x_{i}(t)\right)+\sum_{j=1}^{N} G_{i j} \Gamma x_{j}(t-\tau(t)), \\
i=1, \ldots, N, t \in\left(t_{k-1}, t_{k}\right], \\
x_{i}\left(t_{k}^{+}\right)=c_{i} x_{i}\left(t_{k}\right)+\left(1-\sigma_{i}\left(t_{k}\right)\right) K_{i} x_{i}\left(t_{k}\right) \\
+\sigma_{i}\left(t_{k}\right) K_{i} \bar{x}_{i}\left(t_{k-1}\right), \quad t=t_{k}^{+}, k=1,2, \ldots, \\
x_{i}(t)=\varphi_{i}(t), \quad t \in[-\bar{\tau}, 0] .
\end{gathered}
$$

The parameters are given as follows:

$$
\begin{aligned}
E & =\left[\begin{array}{llll}
1 & 0 & 0 & 0 \\
0 & 1 & 0 & 0 \\
0 & 0 & 1 & 0 \\
0 & 0 & 0 & 0
\end{array}\right], \quad c_{1}=c_{2}=1.2, \\
A & =\left[\begin{array}{rrrr}
-10 & 10 & 0 & 0 \\
\frac{8}{3} & -1 & 0 & 0 \\
0 & 0 & \frac{-8}{3} & 0 \\
0 & 0 & 2 & 1
\end{array}\right], \quad N=2, \\
f\left(t, x_{i}(t)\right) & =\left(0\left(-x_{i 1}(t) x_{i 3}(t)\right)^{T}\left(x_{i 1}(t) x_{i 2}(t)\right)^{T} 2 x_{i 3}(t)\right)^{T} .
\end{aligned}
$$

For simplicity, consider the system with 2 nodes. Assume that the external coupling matrix is $G=\left[\begin{array}{cc}-7 & 3 \\ 3 & -4\end{array}\right]$ and the internal coupling matrix is

$$
\Gamma=\left[\begin{array}{cccc}
0.1 & -0.2 & -0.1 & 0 \\
0 & 0.1 & 0.2 & 0 \\
-0.2 & 0 & 0.1 & 0 \\
0.1 & 0.2 & 0.1 & -0.1
\end{array}\right]
$$

Supposing that $\tau(t)=0.02$ sint. According to Lemma 1, we can choose $P=I_{8 \times 8}, T=t_{k+1}-t_{k}=0.005$. The region of parameters of chaotic system is $L=80$, and the gain of impulsive controller is $-1.8653 \leq K \leq-0.5347$. The state trajectory diagram of system is depicted in Figure 1. For the case of packet dropouts probability is $\operatorname{Pr}(\sigma(k)=0 \mid 0.8)$ and initial condition is $x_{1}(t)=\left[\begin{array}{llll}3 & 2 & -1 & 2\end{array}\right]^{T}, x_{2}(t)=$ $\left[\begin{array}{llll}6 & 5 & -4 & 8\end{array}\right]^{T}$, and $t \in(-\tau, 0)$.

Figure 1 shows that the asymptotic stability of the closedloop uncertain system can be guaranteed using the networked impulsive controller designed in this paper. 


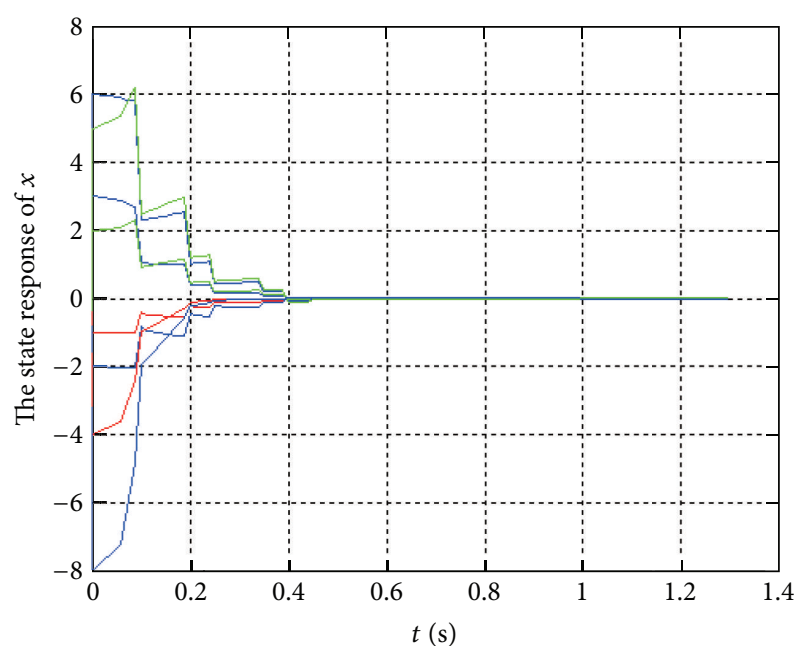

FIGURE 1: The state response of CNSINCS via impulsive control (color online).

\section{Conclusion}

In this paper, the global exponential stability CNSINCS via impulsive control is investigated. According to the Lyapunov stability theory, the mathematic model of CNSINCS is established. A general model of network consisting of time-delay and packet dropouts has been formulated and the globally exponential stable sufficient conditions have been established. Impulsive controller, which may ensure the system achieves exponential stability with a given decay rate is designed. Therefore our control scheme is efficient and practical in dealing with problems of data transmission with time-delay and packet dropouts. As an application, a numerical simulation is given to demonstrate the usefulness and practicability of proposed theoretical results.

\section{Acknowledgments}

This work was supported by the Jiangsu Planned Projects for Postdoctoral Research Funds under Grants 1202005C and the Jiangsu Natural Science Funds for Young Scholar under Grants BK20130696.

\section{References}

[1] D. Yang, C. Sha, and Q. Zhang, " $H_{2}$ analysis and parameterized $\mathrm{H}_{2}$ observer design for descriptor systems," in Proceedings of the 6th World Congress on Intelligent Control and Automation (WCICA '06), vol. 1, pp. 2370-2374, Dalian, China, June 2006.

[2] S. Q. Zhu and N. Ji, Robust Control for Linear Singular TimeDelay Systems, School of Mathmetics, Shandong University, 2005.

[3] T. Li, L. Guo, and L. Wu, "Simplified approach to the asymptotical stability of linear systems with interval time-varying delay," IET Control Theory and Applications, vol. 3, no. 2, pp. 252-260, 2009.

[4] T. Li and W. X. Zheng, "Networked-based generalised $H_{\infty}$ fault detection filtering for sensor faults," International Journal of Systems Science, 2013.
[5] J. Lin and S. Fei, "Reliable control for a class of uncertain singular systems with interval time-varying delay," Asian Journal of Control, vol. 13, no. 4, pp. 542-552, 2011.

[6] B. Sfaihi and Mo. Benrejeb, "On stability analysis of nonlinear discrete singularly perturbed T-S fuzzy models," International Journal of Dynamics and Control, vol. 1, no. 1, pp. 20-31, 2013.

[7] J. N. Li, H. Y. Su, Y. B. Zhang, Z. G. Wu, and J. Chu, "Chattering free sliding mode control for uncertain discrete time-delay singular systems," Asian Journal of Control, vol. 15, no. 1, pp. 260-269, 2013.

[8] X. Sun, Q.-L. Zhang, C.-Y. Yang, Z. Su, and Y.-Y. Shao, "An improved approach to delay-dependent robust stabilization for uncertain singular time-delay systems," International Journal of Automation and Computing, vol. 7, no. 2, pp. 205-212, 2010.

[9] J. A. K. Suykens, T. Yang, and J. L. O. Vandewalle, "Impulsive control and synchronization of chaos," in Controlling Chaos and Bifurcations in Engineering Systems, G. Chen, Ed., pp. 275-298, CRC Press, Boca Raton, Fla, USA, 1999.

[10] Z.-H. Guan, J. Huang, and G. Chen, "Stability analysis of networked impulsive control systems," in Proceedings of the 25th Chinese Control Conference (CCC '06), pp. 2041-2044, Harbin, China, August 2006.

[11] J. Yao, Z.-H. Guan, G. Chen, and D. W. C. Ho, "Stability, robust stabilization and $H_{\infty}$ control of singular-impulsive systems via switching control," Systems and Control Letters, vol. 55, no. 11, pp. 879-886, 2006.

[12] T. Yang, "Impulsive control," IEEE Transactions on Automatic Control, vol. 44, no. 5, pp. 1081-1083, 1999.

[13] J. Sun, Y. Zhang, and Q. Wu, "Less conservative conditions for asymptotic stability of impulsive control systems," IEEE Transactions on Automatic Control, vol. 48, no. 5, pp. 829-831, 2003.

[14] H. Ye, A. N. Michel, and L. Hou, "Stability theory for hybrid dynamical Systems," IEEE Transactions on Automatic Control, vol. 43, no. 4, pp. 461-474, 1998.

[15] T. Yang, Impulsive Control Theory, Springer, Berlin, Germany, 2001.

[16] Y. Dai, Y. Cai, and X. Xu, "Synchronisation analysis and impulsive control of complex networks with coupling delays," IET Control Theory and Applications, vol. 3, no. 9, pp. 1167-1174, 2009. 


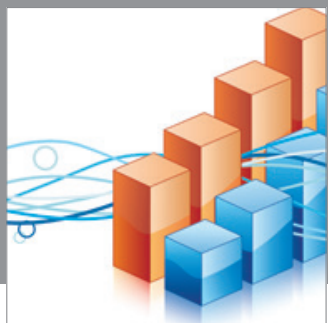

Advances in

Operations Research

mansans

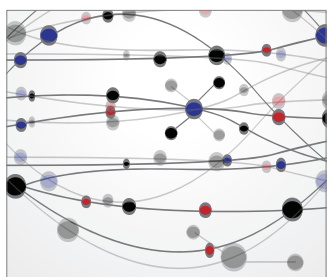

The Scientific World Journal
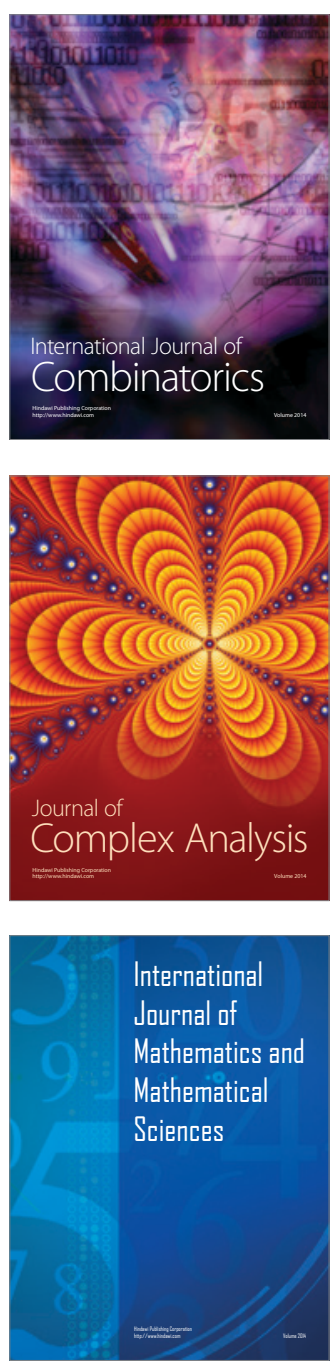
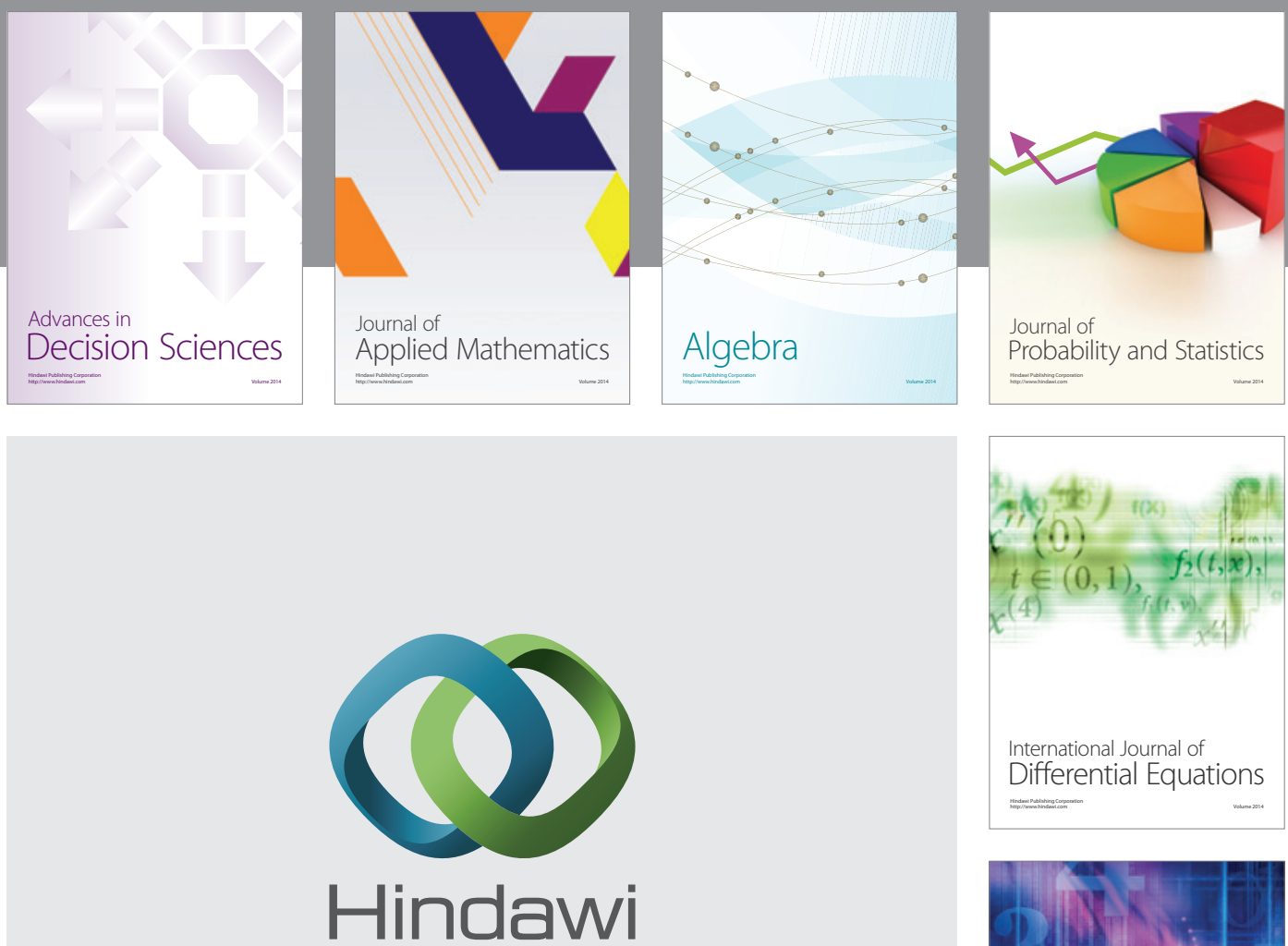

Submit your manuscripts at http://www.hindawi.com
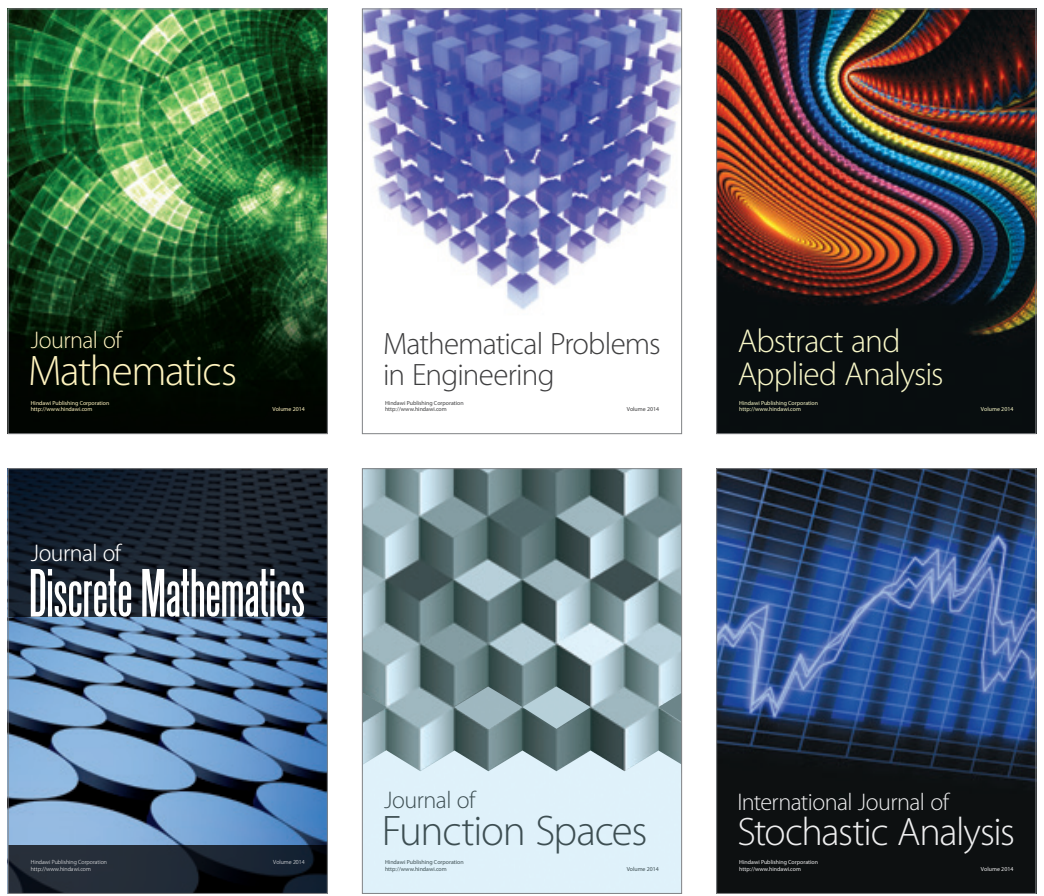

Journal of

Function Spaces

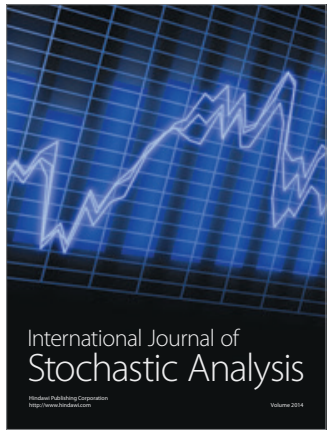

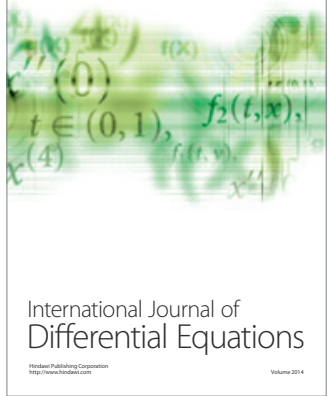
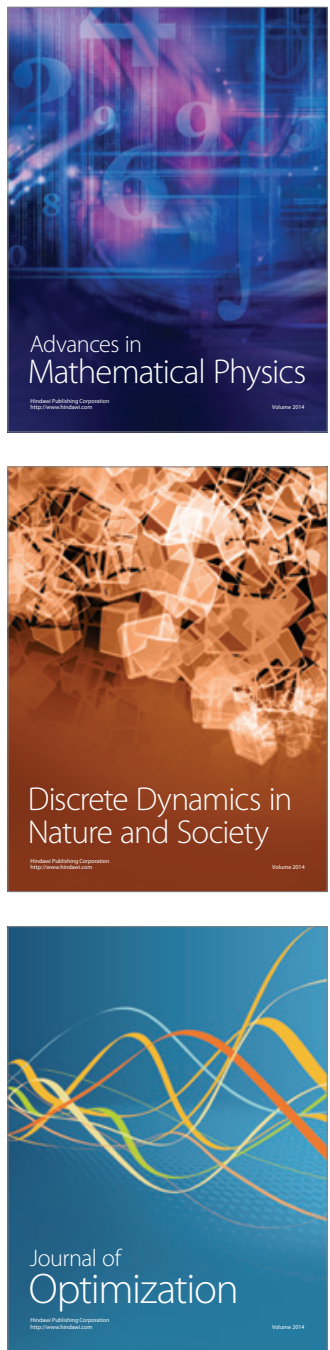